\title{
The landscape of alternative splicing in HIV-1 infected CD4 T-cells
}

\author{
Seyoun Byun ${ }^{1 \dagger}$, Seonggyun Han $^{1+}$, Yue Zheng ${ }^{2}$, Vicente Planelles ${ }^{2}$ and Younghee Lee ${ }^{1,3^{*}}$ \\ From The International Conference on Intelligent Biology and Medicine (ICIBM) 2019 \\ Columbus, OH, USA. 9-11 June 2019
}

\begin{abstract}
Background: Elucidating molecular mechanisms that are altered during HIV-1 infection may provide a better understanding of the HIV-1 life cycle and how it interacts with infected T-cells. One such mechanism is alternative splicing (AS), which has been studied for HIV-1 itself, but no systematic analysis has yet been performed on infected T-cells. We hypothesized that AS patterns in infected T-cells may illuminate the molecular mechanisms underlying HIV-1 infection and identify candidate molecular markers for specifically targeting infected T-cells.

Methods: We downloaded previously published raw RNA-seq data obtained from HIV-1 infected and non-infected T-cells. We estimated percent spliced in (PSI) levels for each AS exon, then identified differential AS events in the infected cells (FDR $<0.05, \mathrm{PSI}$ difference $>0.1$ ). We performed functional gene set enrichment analysis on the genes with differentially expressed AS exons to identify their functional roles. In addition, we used RT-PCR to validate differential alternative splicing events in cyclin T1 (CCNT1) as a case study.

Results: We identified 427 candidate genes with differentially expressed AS exons in infected T-cells, including 20 genes related to cell surface, 35 to kinases, and 121 to immune-related genes. In addition, protein-protein interaction analysis identified six essential subnetworks related to the viral life cycle, including Transcriptional regulation by TP53, Class I MHC mediated antigen, G2/M transition, and late phase of HIV life cycle. CCNT1 exon 7 was more frequently skipped in infected T-cells, leading to loss of the key Cyclin_N motif and affecting HIV-1 transcriptional elongation.

Conclusions: Our findings may provide new insight into systemic host AS regulation under HIV-1 infection and may provide useful initial candidates for the discovery of new markers for specifically targeting infected T-cells.
\end{abstract}

Keywords: Alternative splicing, CCNT1, HIV-1, CD46, CD4 T-cell

\section{Background}

Current treatments for HIV patients (e.g. highly active antiretroviral therapy, HAART) mainly aim to effectively suppress viral load, recover immunologic function, and prevent HIV from acquiring drug-resistant mutations [1-3]. Such treatments rely on preventing the infection from progressing to active AIDS, thereby improving survival. The complete elimination of the infection would

\footnotetext{
* Correspondence: younghee.lee@utah.edu

tSeyoun Byun and Seonggyun Han contributed equally to this work. ${ }^{1}$ Department of Biomedical Informatics, University of Utah School of Medicine, Salt Lake City, UT, USA

${ }^{3}$ Huntsman Cancer Institute, University of Utah School of Medicine, Salt Lake City, UT, USA

Full list of author information is available at the end of the article
}

be ideal, but is complicated by virus harbored in T-cells (i.e. provirus) [4]. Since the virus relies on the molecular machinery of the host $\mathrm{T}$-cell for its replicative cycle during infection [4-8], understanding biological host factors involved in the viral life cycle may provide us with a basis for future drug development.

Genome-wide investigation of molecular changes in infected T-cells is a promising approach by which to identify a better strategy for curing HIV patients [9]. For example, genome-wide gene expression and DNA methylation profiling have successfully demonstrated changes in the molecular patterns of HIV-infected cells [9-12]. One such change occurs for nuclear factor IX (NFIX), which showed lower histone methylation in Tcells infected with HIV-1, consequently leading to its 
increased expression [11]. This increased NFIX may interact with the core-negative regulatory element (NRE) in the HIV-1 long terminal repeat (LTR), thus inhibiting the transcription of HIV-1.

Another potential mechanism by which HIV may regulate the molecular machinery of infected T-cells is alternative splicing (AS). Various immune cells use AS to regulate their proper activation or inactivation [13, 14]. Over $60 \%$ of genes produce immune cell-specific (i.e. T- or B-cell) AS isoforms $[15,16]$, and many such genes have been experimentally validated as relevant to immune responses, including cell surface receptors, cytokine-related genes, transcription regulators, RNA processing genes, intracellular signaling/transport genes, ion channels, and cytoskeletal genes [17]. For example, Fas cell surface death receptor $(F A S)$ produces transcript isoforms with exon 6 skipping during peripheral blood mononuclear cell activation [18]. Another example is protein tyrosine phosphatase, receptor type C (CD45), in which skipping of exon 6 regulates immune signaling via protein kinase C (PKC) and Ras [17, 19]. A previous study carried out an exon array-based investigation of the transcriptome in uninfected and HIV-infected CD4 T-cells at $24 \mathrm{~h}, 48 \mathrm{~h}$, and $72 \mathrm{~h}$ post-infection [4]. They reported that exon 54 of Inositol 1,4,5-Trisphosphate Receptor Type 1 (ITPR1), an ion channel that plays a role in lymphocyte activation, was skipped in HIV-1 infected T-cells. This suggests that a genome-wide scan of AS events in HIVinfected T-cells has the potential to identify additional molecular signatures of HIV-1 infection.

Here, we analyzed RNA-seq data from infected and non-infected T-cells, originally collected by B. Descours et al. [9]. We identified 427 genes that have differential splicing events in T-cells infected by HIV-1. These genes were significantly enriched for HIV replication-related pathways. Furthermore, as a case study, we experimentally validated an exon of cyclin T1 (CCNT1) that had increased skipping in infected T-cells; this and other splicing events could provide potential biomarkers for capturing infected T-cells. Our study provides not only additional insight into AS events as an underlying biological mechanism of HIV-1 infection, but also the opportunity to explore novel biomarkers for detecting infected T-cells.

\section{Methods}

Additional file 1: Figure. S1 illustrates the overall pipeline of RNA-seq data analysis by which alternative splicing events were identified between infected and uninfected T-cells, along with functional interpretation of the alternative splicing events.

\section{Data and pre-processing}

We obtained RNA-seq data (i.e. FASTQ files) from the NCBI Sequence Read Archive (SRA) (https://www.ncbi. nlm.nih.gov/sra, SRP id = SRP094482 and run ids = SRR5071107 - SRR5071122) [9]. The data was comprised of four sample groups: $\mathrm{XH}+, \mathrm{Vpx}$-treated infected T-cells indicating green fluorescent protein (GFP) $(n=$ 4); XH-, Vpx-treated T-cells exposed to HIV, but HIVnegative T-cells $(n=4) ; \mathrm{X}, \mathrm{Vpx}$-treated T-cells not exposed to HIV $(n=4)$; and NI, non-infected T-cells with neither Vpx treatment nor HIV exposure $(n=4)$ (Additional file 1: Figure S1, Step 1). The raw FASTQ file contained 75 100 million single-end reads of 101 nucleotides in length per sample. We performed general quality control, including evaluation of the reads quality and average GC content in each base, of whole reads using the FastQC package v0.11.4 [20] (Additional file 1: Figure S1, Step 2). We then removed contaminants that may have originated from adaptor or linker sequences using Trimmomatic v0.32 [21]. After this preprocessing, we mapped the reads to the human reference genome (GRCH37.75 based on hg19 reference sequence) using TopHat v2.1.1 [22]. All samples had unique mapping rates of approximately $80 \%$. Among the multiple mapped reads in our significant 494 AS events, approximate $84 \%$ mapped to conserved regions. The remainder of multiple mapped reads account for only $0.66 \%$ of mapped reads that was used for determining the 494. AS events.

\section{Identification of AS events by RNA-seq}

We identified AS events in junction reads using rMATs v3.2.5 [23]. We considered all possible AS events: exon skipping (ES), alternative $5^{\prime}$ splicing site (A5SS), alternative 3' splicing site (A3SS), intron retention (IR), and mutually exclusive exons (MXE). The rMATs was used to filter out multiple mapped reads, and reads spanning exon-exon junctions (junction reads) were used for identifying ES, A5SS, A3SS, and MXE. Also, for estimation of IR, rMATs used reads spanning exon-exon junctions and in retained introns. We estimated the expression level of each AS event, denoted as percent spliced in (PSI), the fraction of alternatively spliced exons estimated from exon-exon junction reads (Additional file 1: Figure S1, Step 3). A PSI value of zero means that all mRNAs in a given gene exclude the given exon, while a value of 1 indicates that the given exon is never skipped (i.e., a constitutive exon). We first compared the expression of each identified AS event between the case group of HIV-1 infected T-cells and each control group of non-infected T-cells (i.e., $\mathrm{XH}+$ vs. $\mathrm{XH}-, \mathrm{XH}+$ vs. $\mathrm{X}$, and $\mathrm{XH}+$ vs.NI) and collected the union of differentially expressed AS events from each comparison. Next, To obtain a pure set of differentially expressed AS exons between case and controls, we performed additional comparisons between every possible pair of controls (i.e., XH- vs. X, XH- vs. NI, and X vs. NI). We then excluded differential AS exons that were also identified in these control group comparisons from the union of case events (Fig. 1a). An AS event was considered significant if it 


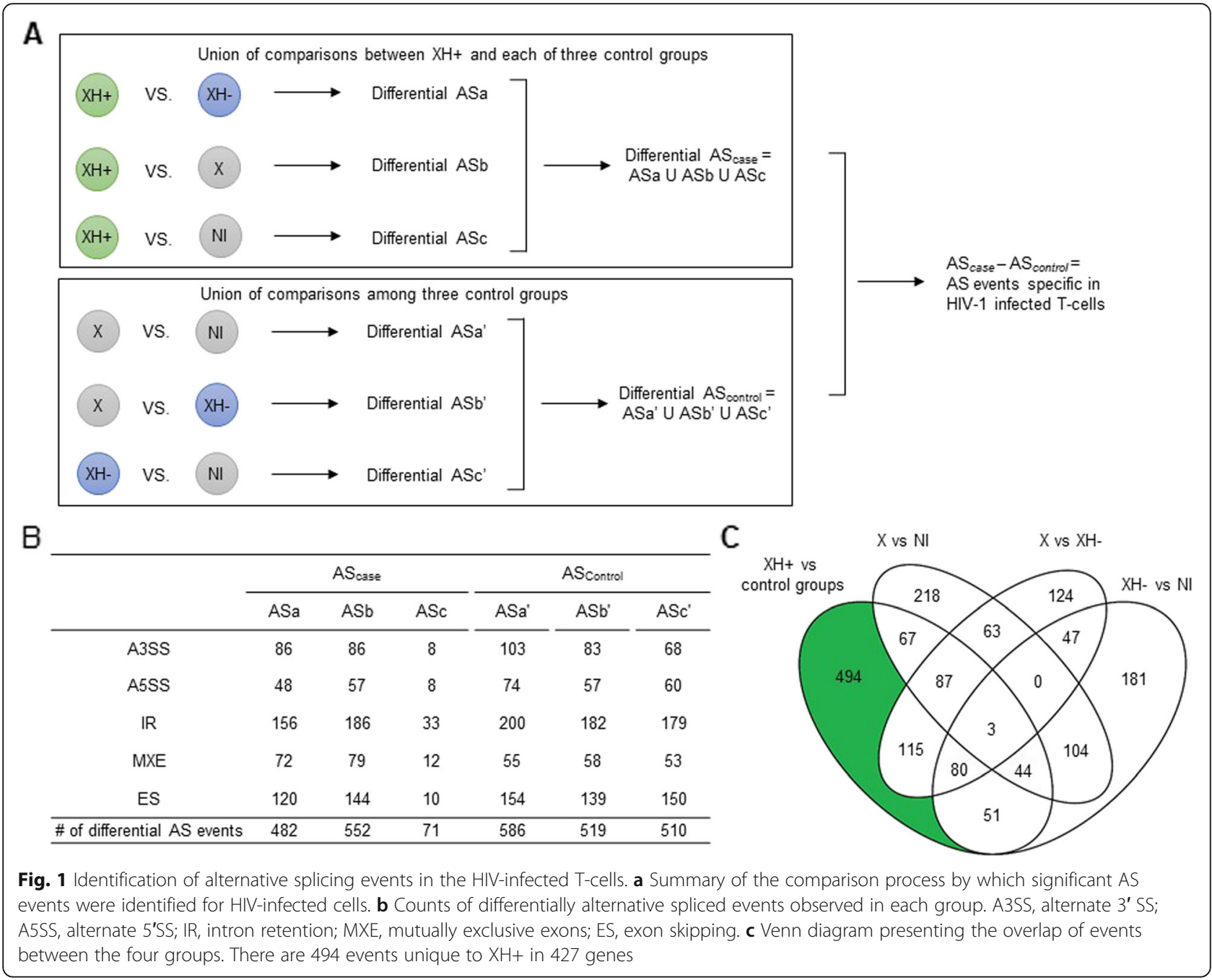

had FDR-corrected $p$-value $<0.05$ and PSI $>0.1$, i.e. $>10 \%$ difference in expression between groups.

\section{Gene set enrichment analysis}

For the functional interpretation of identified AS genes, we conducted gene set enrichment analysis over canonical pathways and Gene Ontology (GO) terms using ConsensusPathDB v32 (CPDB; http://cpdb.molgen.mpg.de/) [24]. Pathways and terms were considered significant at FDR < 0.05 . To reduce the redundancy of GO terms, we limited the GO hierarchy level from two to four categories and filtered out potentially false positive GO terms using GO-Module v1.0 [25]. In addition, for GO terms with similar functions, we then selected only the most significant term (Additional file 1: Figure S1, Step 4).

\section{Network analysis}

We constructed a protein-protein interaction network for the set of identified AS genes using StringDB v10.5 [26] (Additional file 1: Figure S1, Step 4). We selected interaction relationships between genes with the highest confidence $>0.9$ and without disconnected nodes in the network.

\section{Visualization of protein 3D structure}

As a case study for the functional impact of AS, we investigated the functional impacts of AS events identified in two genes, CCNT1 and the CD46 molecule (CD46), on their protein domains and 3D structures. To determine the alteration of CCNT1 protein structure, we obtained the X-ray crystallography structure of its canonical protein product (PDB id: 5l1z) from the Protein Data Bank [27]. Protein 3D structures were visualized with PyMol v1.3 [28] (Additional file 1: Figure S1, Step 4).

\section{Experimental validation of CCNT1}

Cell culture: HEK293 cells were cultured in DMEM (Gibco, US) containing 10\% FBS (Atlanta, US), 2 mM LGlu (Gibco, US). Primary cells were isolated from peripheral blood from healthy donors, obtained by venipuncture. 
Primary cells were cultured in RPMI1640 (Gibco, US) containing 10\% FBS, $2 \mathrm{mML}-\mathrm{Glu}$ and $30 \mathrm{IU} / \mathrm{ml} \mathrm{IL}-2$ (Stem Cell Technologies, US). $\mathrm{R}^{2}=0.03$.

Virus production: Pseudotype viruses, pNL4.3-deltaEnv-nLuc-2ANef -VSVG, were produced by cotransfecting pNL4.3-deltaEnv-nLuc-2ANef (produced by Laura Martins in our lab) and pCMV-VSVG (Addgene) into HEK293 cells. After 2 days, cell supernatant was collected and filtered with a 0.22 um filter. Viruses were titrated on SupT1 cells and stored at $-80^{\circ} \mathrm{C}$.

HIV latency assay: TCM cells were generated and cultured as previously described (Bosque A, Planelles V. Induction of HIV-1 latency and reactivation in primary memory CD4+ cell. Blood 2009, 113:58-65). Briefly, naïve cells from heathy donor were activated by antihuman IL-4 (Peprotech, US), anti-human IL-12 (Peprotech, US), TGF- $\beta 1$ (Peprotech, US) and Dynabeads CD3/ CD28 (Invitrogen, US) for 3 days. At Day 4, beads were removed and activated cells were cultured in fresh medium. At Day 7, cells were infected by pseudotyped pNL4.3-deltaEnv-nLuc-2ANef -VSVG. At Day 10, CD4 and P24 levels were checked by flow cytometry. CD4+ cells were isolated by a magnet kit (ThermoFisher Scientific, US). At Day 12, uninfected cells, CD4+ cells and CD4- were collected for qPCR test. At Day 20, uninfected cells and CD4+ cells were collected for qPCR test.

qPCR: RNA from cell samples were isolated according to the manual procedure of RNease Mini Kit (Qiagen, US). Reverse transcription was done using SSIV First Strand Synthesis System (Invitrogen, US). qPCR was done using Platinum SYBR Green qPCR SuperMix (Invitrogen, US).

Primers for CCNT1a: (Forward) AACAGCCTGC ATTTGACCAC and (Reverse) ATCTCCCAATTGGA CCACTTG. Primers for CCNT1b: (Forward) CAACCA ACAGAACTGACACTG and (Reverse) ATCTGTTCCT CGGTCATCTG. Program on LightCycler480 (Roche, US): step 1: $50^{\circ} \mathrm{C} 2 \mathrm{~min}$. Step 2: $95^{\circ} \mathrm{C} 2 \mathrm{~min}$. Step 3: 45 cycles of $95^{\circ} \mathrm{C} 30 \mathrm{~s}, 56^{\circ} \mathrm{C} 60 \mathrm{~s}$ and $72^{\circ} \mathrm{C} 30 \mathrm{~s}$.

\section{Results}

As described in the Methods, we conducted multiple comparisons to identify the set of differential AS events particular to HIV-1 infected T-cells. We identified 482, 552 , and 71 statistically significant AS events $(F D R<$ 0.05 and PSI $>0.1$ ) in three case-control comparisons, $\mathrm{XH}+$ vs. $\mathrm{XH}-, \mathrm{XH}+$ vs. $\mathrm{X}$, and $\mathrm{XH}+$ vs. NI, respectively (see the Methods and Fig. 1a); these collectively comprised 941 AS events across 751 genes (Fig. 1b). After exclusion of 447 statistically significant AS events (379 genes) that were also detected in at least one of three control group comparisons (Fig. 1c), we were left with 494 AS events (427 genes). This set of 494 AS events (427 genes, including 20 cell surface-related, 35 kinase, and 121 immune-related genes) were considered to be uniquely differentially expressed in infected T-cells. The results are summarized in Additional file 2: Table S1.

\section{AS genes are enriched in pathways relevant to the HIV-1 life cycle}

To gain insight into the molecular functions of the 427 AS genes and their roles in the HIV-1 life cycle, we performed gene set enrichment analysis for canonical pathways and Gene Ontology (GO) terms using CPDB (see the Methods). After reducing redundant terms, we identified 23 significantly enriched pathways $(F D R<0.05$; Additional file 3: Table S2, Additional file 1: Figure S2). These included pathways related to the late phase of HIV-1 infection and transcriptional reactivation of HIV, such as "Late Phase of HIV Life Cycle," "HIV Transcription Initiation," "Formation of HIV-1 Elongation Complex Containing HIV-1 Tat," "Transcriptional Regulation by TP53," "Clathrin Derived Vesicle Budding," and "Membrane Trafficking." The "Late Phase of HIV Life Cycle" pathway encompasses processes spanning HIV-1 transcriptional activation through budding and maturation, including HIV-1 intracellular trafficking and assembly [29]. The TP53 pathway is well-known to negatively regulate HIV-1 transcription by inactivating its long terminal repeat (LTR) promoter. Membrane trafficking is also an important pathway for the recruitment and assembly of proteins required for HIV-1 budding. These results suggest that our 427 AS genes are implicated in HIV-1 replication in T-cells, and that there is a need to elucidate differential AS underlying host molecular mechanisms affecting HIV-1 genome replication (i.e. activation of HIV-1 transcription, assembly, and budding).

Within the set of 427 AS genes, 21 non-redundant GO terms were additionally overrepresented $($ FDR $<0.05)$, including "positive regulation of immune effector process" (GO:0002699), "regulation of cell cycle" (GO: 0051726), and "IkappaB kinase activity" (GO:0008384) (Additional file 4: Table S3, Additional file 1: Figure S3). Enrichment in immune response-related GO terms was expected. Genes in these terms regulate cell cycle arrest and stimulate HIV-1 LTR-driven transcription [30, 31] in the infected T-cells. Meanwhile, IkappaB kinase is one of the key regulators of viral replication, in which an AS event may produce a novel inhibitor of HIV-1 replication [32]. Therefore, the enrichment analysis suggests a potential for AS to play a role in the HIV-1 infection within infected $\mathrm{T}$-cells by modulating immune-related genes and pathways related to HIV-1 replication.

\section{Protein interaction network}

To gain further insight into the molecular signature of AS events related to HIV-1 infection, we constructed a 
protein-protein interaction network for the 427 identified AS genes. We then superimposed the significantly enriched canonical pathways and GO terms onto the network, identifying topological neighbors. At the network level, topological distance refers to the functional similarity of two proteins; topologically-neighboring genes are likely to have the same or similar functions [33]. In this analysis, our 427 AS genes functionally grouped into six subnetworks related to HIV-1 infection in T-cells, including regulation by TP53, vesiclemediated transport, late phase of HIV life cycle, G2/M transition, and Class I MHC mediated antigen (Fig. 2). As mentioned above, TP53, vesicle-mediated transport, and late phase of HIV life cycle pathways all play functional roles in HIV-1 replication in T-cells; meanwhile, regulation of the G2/M transition pathway by HIV-1 viral infectivity factor induces G2 cell cycle arrest [34]. Class I MHC mediated antigen genes are also related to immune system function in infected T-cells. Finally, we created a schematic of the HIV-1 life cycle in T-cells that summarizes its overall modulation by AS (Fig. 3).

\section{Case study}

As described above, our AS genes were enriched in pathways related to HIV-1 replication. In other words, the modulation of these genes by AS may be important for HIV-1 replication. As a case study, we selected a gene for functional analysis, CCNT1. We present further analysis and discussion of this gene's potential role as a modulator of HIV-1 transcription and a marker specific to infected T-cells.

CCNT1 is a member of the cyclin c subfamily and a component of the positive transcription elongation factor b (P-TEFb) complex with CDK9 and tat, making it essential for transcriptional elongation from the HIV-1 viral genome $[35,36]$. In this study, we recapitulated a previously-identified exon 7 skipping event (164 base pairs) that may introduce an early stop codon, resulting in a potentially truncated protein or one subject to nonsense-mediated decay [37] (Fig. 4a). Exon 7 tends to be more skipped in HIV-1 infected T-cells than in noninfected $\left(F D R=3.10 \times 10^{-10}\right)$ (Fig. $\left.4 \mathrm{~b}\right)$, which we validated experimentally (Fig. 5). As described in Fig. 4a, skipping of CCNT1 exon 7 prevents it from encodes the Cyclin_N domain (PF00134); a protein that lacks this domain due to exon skipping may be unable to participate in the P-TEFb complex with Tat [38]. Figure 4c shows the $3 \mathrm{D}$ protein structure with the affected region highlighted in purple, adjacent to Tat. Furthermore, CCNT1 function is known to be greatly regulated by alternative splicing $[39,40]$. Therefore, this exon skipping event may be an important factor for inhibiting HIV-1 transcription through repression of the P-TEFb complex.

\section{Discussion}

The paper in which the RNA-seq data analyzed in this study was originally published focused on genes differentially expressed between latently infected and noninfected T-cells. They identified overexpressed CD32a in

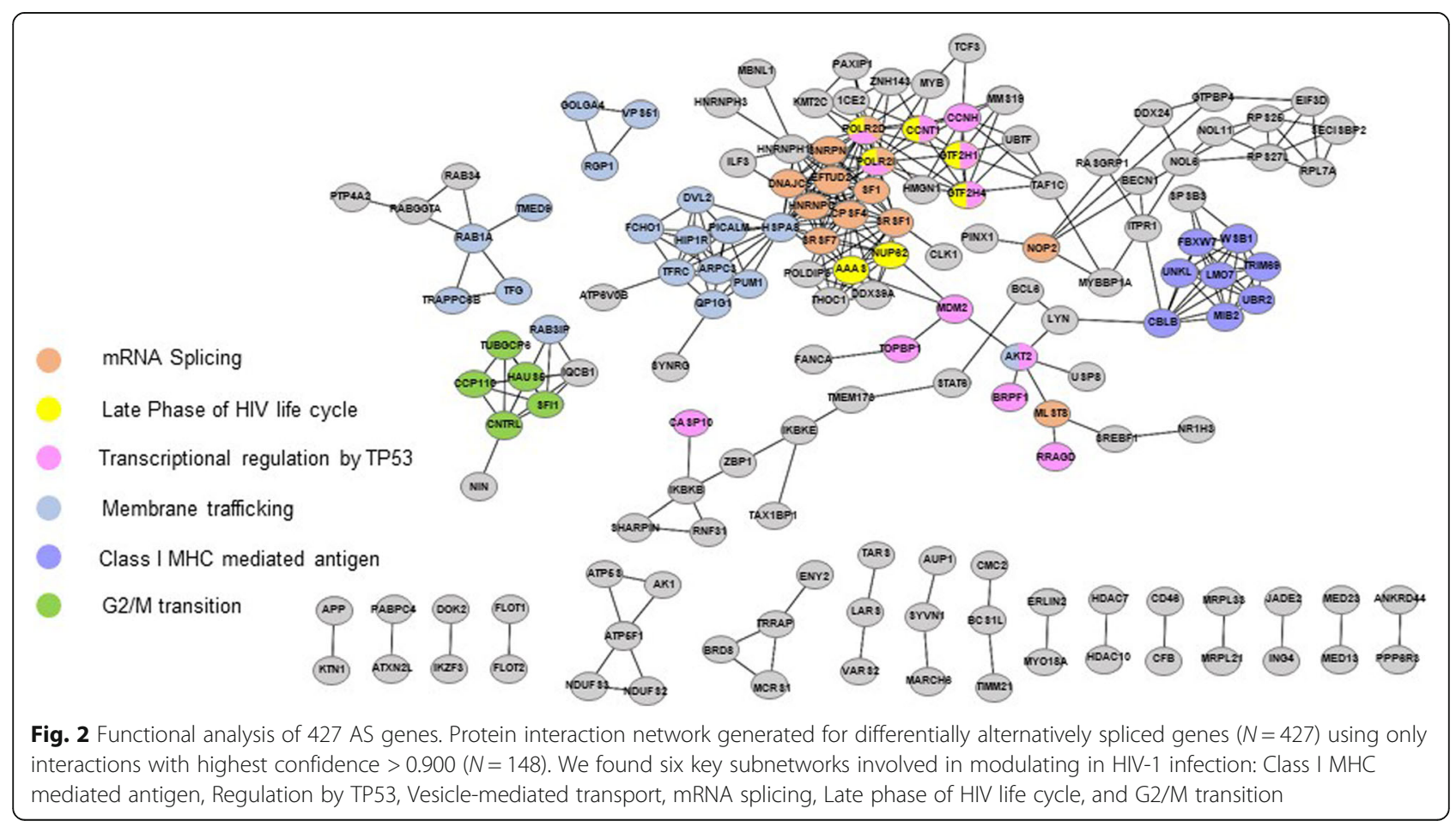




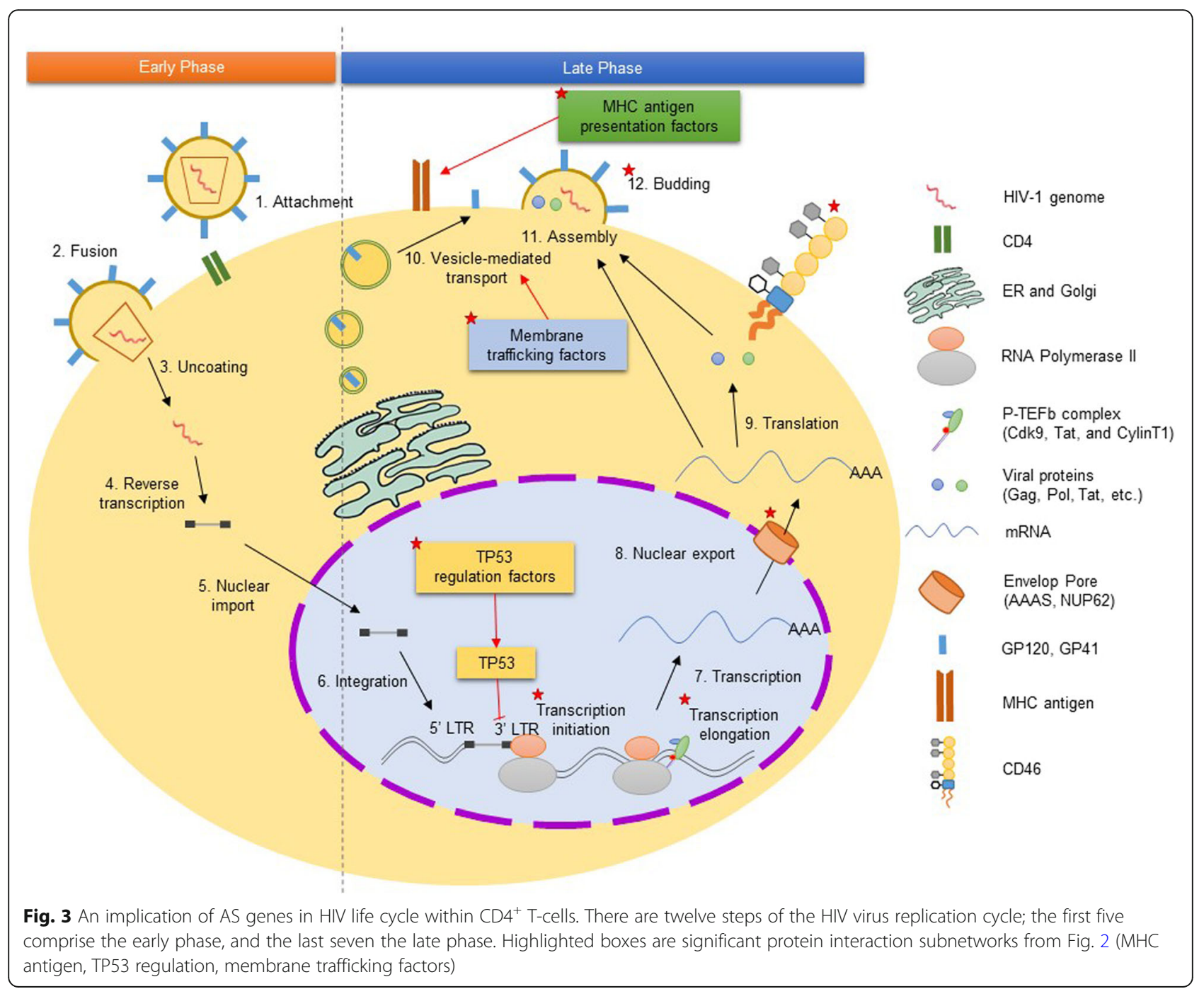

the latently-infected $\mathrm{XH}+$ group, suggesting that $\mathrm{CD} 32 \mathrm{a}$ could be a potential latent-specific biomarker. However, there is an ongoing debate on whether this paper properly distinguished resting T-cells from activated CD4 Tcells [41]. In other words, expression of CD32a may not be solely enriched in resting T-cells [42, 43]. While there is an additional issue in the original study's definition of the latently-infected $\mathrm{XH}+$ group, this group can at least be confidently defined as infected because integration of the HIV-1 genome was confirmed by GFP [9]. Therefore, in this study we focus on infected CD4 T-cells.

Meanwhile, we experimentally validated that exon 7 of CCNT1 was more frequently skipped in T-cells confirmed to be latently infected (Fig. 5 and Additional file 1: Figure S4) in our experimental system (see the Methods). This skipping event may lead to loss of CCNT1 function (Cyclin_N) to interact with Tat (Fig. 4a) $[44,45]$. Notably, CCNT1/CDK9 complex formation with Tat is crucial for activating transcription of the
HIV-1 genome. CCNT1 is one of the P-TEFb complex components, and it plays an essential role in Tatmediated HIV-1 transcription, activating the HIV-1 LTR promoter through direct interaction with Tat protein $[44,45]$. In fact, there is a protein structure assay to identify how the complex specifically interacts with Tat [46]. HIV-1 transcriptional regulation is one of the most important factors in understanding the HIV-1 life cycle during infection, including latency. CCNT1 is a candidate gene that may act as a key factor for controlling transcription of the HIV-1 genome, thus we selected this gene as a case study. Our experiment using RT-PCR suggests that the CCNT1/CDK9 complex is less abundant in latently infected CD4 T-cells, and enriched upon the reactivation of latent HIV-1 [47]. Therefore, our result suggests that exon 7 skipping of CCNT1 may be functionally important for maintaining the latent phase of an HIV-1-infected CD4 T-cell as it leads to the loss of the key domain for P-TEFb complex constitution, 


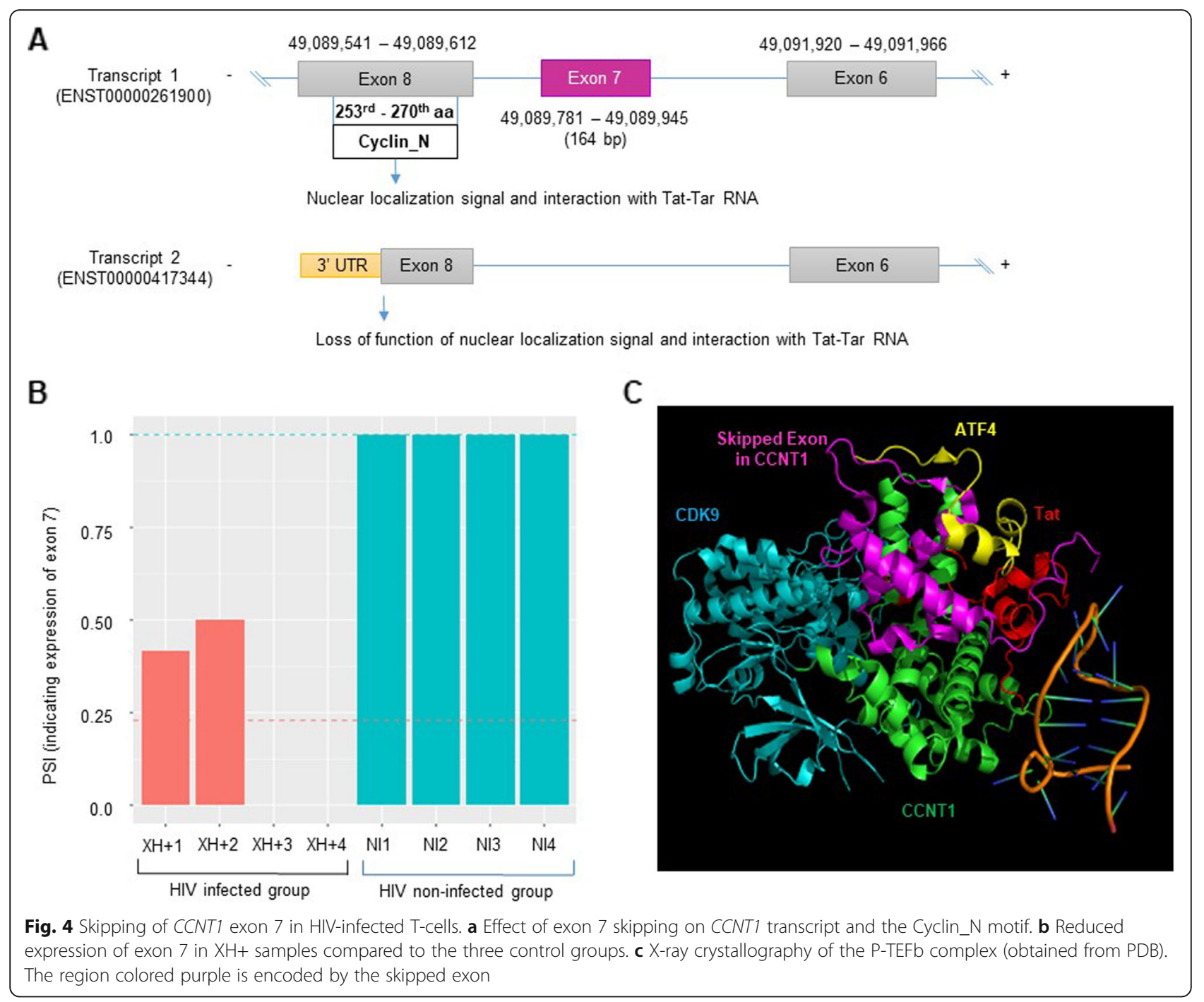

ultimately contributing to the transcriptional inactivation of HIV-1.

To our knowledge, this study is the first of its kind, having been designed to comprehensively explore alternative splicing events in HIV-1-infected T-cells using RNA-seq. We used the rMATS package, which is especially well-designed for replication and group comparison of alternative splicing rates (i.e. PSI values). As observed outcomes may be specific to assembly tools, we conducted a cross-check of our results from rMATS with one of the most popular tools for estimating PSI value, MISO [48]. rMATS outcomes were highly matched with MISO outcomes; the correlation of PSI values between rMATS and MISO for all significant splicing events identified was 0.9 (median correlation). We showed that the 427 identified AS genes were enriched in functional relevance to HIV-1 replication and the HIV-1 infection, suggesting that HIV-1 may be implicated in regulating its host $\mathrm{T}$-cell via alternative splicing.
We constructed the hierarchically clustered heatmap to provide an expression trend of AS exons with PSI values across the cohorts, and $\mathrm{XH}+$ (infected group) samples are clustered closely (Additional file 1: Figure S5). Furthermore, the set of AS genes included genes translated into cell surface proteins, which may provide potential biomarkers for detecting infected T-cells. As an example, CD46 encodes a cell membrane protein that contains four short consensus repeats, a cytoplasmic domain, and a Ser, Thr, Pro-rich region (STP domain). This gene is known to have mutually exclusive exons (exon 7 and 8) (Additional file 1: Figure S6A), and we observed that exon 7 , encoding the STP domain (Additional file 1: Figure S6B), was more likely to be skipped in infected Tcells (Additional file 1: Figure S6C), generating an aberrant protein product that lacks the STP region and may be useable as a marker.

A couple of limitations of the present study needs consideration. Although we presented experimental results 


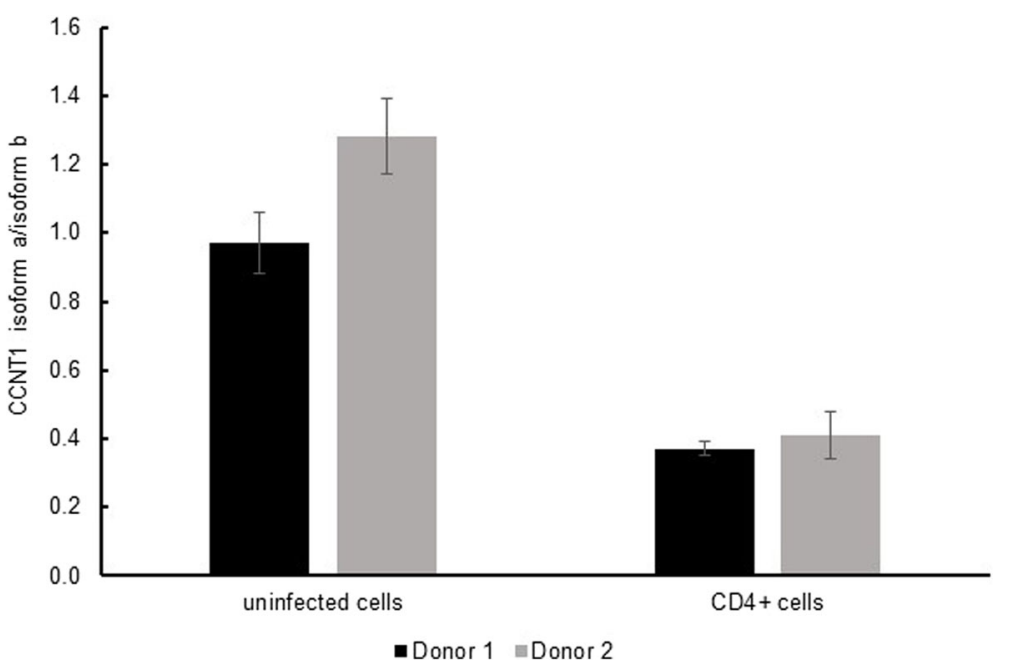

Fig. 5 The ratio of gene expression of CCNT1 isoform a and isoform b. Naïve CD4 cells from healthy donors were isolated and activated. Activated CD4 cells were infected by pseudo-typed HIV viruses for 2 days. At Day20 CD4+ cells containing latently infected cells and uninfected cells were isolated using a magnetic CD4+ isolation kit. Gene expression of CCNT1 isoforms in uninfected cells and CD4+ cells was measured by qPCR. CCNT1 isoform a/isoform b was calculated and shown

from our case study splicing event in CCNT1, we did not validate other AS findings either in vitro or in an independent cohort. Another limitation is the small sample size ( $n=4$ each group). Therefore, further experimental validation or replication with independent data is required from future studies.

\section{Conclusion}

Here, we present the potential of alternatively spliced genes as regulators and biomarkers in infected CD4 Tcells. We conclude that the study of alternative splicing may confer additional understanding of the molecular mechanisms underlying infection of T-cells as well as better treatment strategies for the early elimination of HIV-1.

\section{Supplementary information}

Supplementary information accompanies this paper at https://doi.org/10. 1186/s12920-020-0680-7.

Additional file 1: Figure S1. Overview of AS analysis in HIV-infected and non-infected human primary resting CD4+ T-cells. We obtained RNAseq data for infected and non-infected cells from SRA (reference number SRR5071107-SRR5071122). There are four different treatment groups, and group contained four different cells. We then performed general quality control on the reads, aligned them to the hg19 human reference genome, and selected junction reads to identify alternatively spliced exons. Next, we identified mRNAs with differential alternatively splicing between the case group and each control. The percent spliced in (PSI) ratio indicates the level of differential exon expression. We adjusted q value $<0.05$, and used PSI $>0.01$ as the cutoff for differential splicing. Finally, we performed functional gene set enrichment analysis and classified genes with differential spliced exons in HIV infected T-cells according to their functional roles. Figure S2. 23 canonical pathways enriched in $427 \mathrm{HIV}$ associated AS genes. The red line indicates the cutoff FDR value, $\mathrm{q}<0.05$. Figure S3. $21 \mathrm{GO}$ terms enriched in $427 \mathrm{HIV}$-associated AS genes. GO Biological Process (BP) and Molecular Function (MF) terms were considered significant at an FDR corrected q-value $<0.05$ (red line). Figure $\mathbf{S 4}$.
A plot for providing evidence to select quiescent cells. Ki67 is a proliferation marker, which is not expressed in quiescent cells but is expressed in dividing cells. The graph below shows the ki67 level of cells at Day 19 (red line), which was negative, indicative of lack of cell division. Figure S5. A hierarchically clustered heat map with PSI levels of significant AS events. Groups are annotated by colors in the top of row, and types of AS exons are annotated by colors in the left column. Figure S6. Skipping of CD46 exon 7 skipping in the HIV-infected T-cells. (A) Skipping of exon 7 in transcript 2 and retention of exon 8 (i.e. mutually exclusive exon event) affects the entire STP motif. (B) Location of the STP domain in CD46 protein. (C) Reduced expression of exon 7 in HIV-infected samples. (D) Protein structure of CD46 with the STP domain indicated in purple.

Additional file 2: Table S1. List of AS exons in the infected T-cells. Additional file 3: Table S2. List of AS genes in the enriched canonical pathways.

Additional file 4: Table S3. List of AS genes in the enriched GO terms.

\section{Abbreviations}

A3SS: Alternative 3 ' splicing site; A5SS: Alternative 5' splicing site; AS: Alternative splicing; ES: Exon skipping; FDR: False discovery rate; GFP: Green fluorescent protein; HIV-1: Human immunodeficiency virus 1; IR: Intron retention; MXE: Mutually exclusive exons; PSI: Percent spliced in

\section{Acknowledgments}

The support and resources from the Center for High Performance Computing and Vice President's Clinical and Translational Research Scholar Program at the University of Utah are gratefully acknowledged.

\section{About this supplement}

This article has been published as part of BMC Medical Genomics Volume 13 Supplement 5, 2020: The International Conference on Intelligent Biology and Medicine (ICIBM) 2019: Computational methods and application in medical genomics (part 1). The full contents of the supplement are available online at https://bmcmedgenomics.biomedcentral.com/articles/supplements/ volume-13-supplement-5.

\section{Authors' contributions}

$\mathrm{SB}, \mathrm{SH}$, and $\mathrm{YH}$ conceptualized this study. SB and $\mathrm{SH}$ were involved in the study design and data curations and statistical analyses. YZ and VP performed experimental validation. YH supervised this study. All authors were involved in writing the manuscript and approved it. 


\section{Funding}

The work is supported by development funding from Department of Biomedical Informatics, University of Utah School of Medicine and the Collaborative Genome Program for Fostering New Post-Genome Industry of the National Research Foundation (NRF) funded by the Ministry of Science and ICT (MSIT) (NRF-2017M3C9A6047623). The publication cost is supported by development funding from Department of Biomedical Informatics, University of Utah School of Medicine.

\section{Availability of data and materials}

RNA-seq data (i.e. FASTQ files) used in this study is available in the NCBI Sequence Read Archive (SRA): https://www.ncbi.nlm.nih.gov/sra, SRP id = SRP094482 and run ids = SRR5071107 - SRR5071122.

\section{Ethics approval and consent to participate}

Not applicable.

\section{Consent for publication}

Not applicable.

\section{Competing interests}

The authors declare that the research was conducted in the absence of any commercial or financial relationships that could be construed as a potential conflict of interest.

\section{Author details}

${ }^{1}$ Department of Biomedical Informatics, University of Utah School of Medicine, Salt Lake City, UT, USA. ²Department of Pathology, University of Utah School of Medicine, Salt Lake City, UT, USA. ${ }^{3}$ Huntsman Cancer Institute, University of Utah School of Medicine, Salt Lake City, UT, USA

\section{Published: 3 April 2020}

\section{References}

1. Desai M, lyer G, Dikshit RK. Antiretroviral drugs: critical issues and recent advances. Indian J Pharmacol. 2012;44(3):288-98.

2. Le Douce V, Janossy A, Hallay H, Ali S, Riclet R, Rohr O, Schwartz C. Achieving a cure for HIV infection: do we have reasons to be optimistic? J Antimicrob Chemoth. 2012;67(5):1063-74.

3. Lichterfeld M, Zachary KC. Treating HIV-1 infection: what might the future hold? Ther Adv Chronic Dis. 2011;2(5):293-305.

4. Imbeault M, Giguere K, Ouellet M, Tremblay MJ. Exon level transcriptomic profiling of HIV-1-infected CD4(+) T cells reveals virus-induced genes and host environment favorable for viral replication. PLoS Pathog. 2012;8(8): e1002861.

5. Smith AJ, Li QS, Wietgrefe SW, Schacker TW, Reilly CS, Haase AT. Host genes associated with HIV-1 replication in lymphatic tissue. J Immunol. 2010; 185(9):5417-24.

6. Tasara T, Hottiger MO, Hubscher U. Functional genomics in HIV-1 virus replication: protein-protein interactions as a basis for recruiting the host cell machinery for viral propagation. Biol Chem. 2001;382(7):993-9.

7. Trkola A. HIV-host interactions: vital to the virus and key to its inhibition. Curr Opin Microbiol. 2004;7(4):407-11.

8. Sorin M, Kalpana GV. Dynamics of virus-host interplay in HIV-1 replication. Curr HIV Res. 2006:4(2):117-30.

9. Descours B, Petitjean G, Loez-Zaragoza JL, Bruel T, Raffel R, Psomas C, Reynes J, Lacabaratz C, Levy Y, Schwartz O, et al. CD32a is a marker of a CD4 T-cell HIV reservoir harbouring replication-competent proviruses. Nat. 2017:543(7646):564 +.

10. Yi L, Zhao J, Lu J, Chen Y, Chen L, Cheng J, Sun Y, Li Z, Men R, Yang L, et al. Gene expression profiling of CD4(+) T cells in treatment-naive HIV, HCV mono- or co-infected Chinese. Virol J. 2014;11:27.

11. Kim KC, Lee S, Son J, Shin Y, Yoon CH, Kang C, Choi BS. Identification of novel genes associated with HIV-1 latency by analysis of histone modifications. Hum Genomics. 2017;11(1):9.

12. Corbeil J, Sheeter D, Genini D, Rought S, Leoni L, Du P, Ferguson M, Masys DR, Welsh JB, Fink JL, et al. Temporal gene regulation during HIV-1 infection of human CD4+ T cells. Genome Res. 2001;11(7):1198-204.

13. Yabas M, Elliott H, Hoyne GF. The Role of Alternative Splicing in the Control of Immune Homeostasis and Cellular Differentiation. Int J Mol Sci. 2015: $17(1)$.
14. Schaub A, Glasmacher E. Splicing in immune cells-mechanistic insights and emerging topics. Int Immunol. 2017;29(4):173-81.

15. Grigoryev YA, Kurian SM, Nakorchevskiy AA, Burke JP, Campbell D, Head SR, Deng J, Kantor AB, Yates JR 3rd, Salomon DR. Genome-wide analysis of immune activation in human $T$ and $B$ cells reveals distinct classes of alternatively spliced genes. PLoS One. 2009:4(11):e7906.

16. Ergun A, Doran G, Costello JC, Paik HH, Collins JJ, Mathis D, Benoist C, ImmGen C. Differential splicing across immune system lineages. Proc Natl Acad Sci U S A. 2013;110(35):14324-9.

17. Martinez NM, Lynch KW. Control of alternative splicing in immune responses: many regulators, many predictions, much still to learn. Immunol Rev. 2013;253(1):216-36

18. Liu CD, Cheng JH, Mountz JD. Differential expression of human Fas messenger-Rna species upon peripheral-blood mononuclear cell activation. Biochem J. 1995:310:957-63.

19. Lynch KW, Weiss A. A model system for activation-induced alternative splicing of CD45 pre-mRNA in T cells implicates protein kinase $C$ and Ras. Mol Cell Biol. 2000;20(1):70-80.

20. S A: FastQC: a quality control tool for high throughput sequence data. Available online at: http://www.bioinformatics.babraham.ac.uk/projects/ fastqc. Accessed on 04.27.2017. 2010.

21. Bolger AM, Lohse M, Usadel B. Trimmomatic: a flexible trimmer for Illumina sequence data. Bioinformatics. 2014;30(15):2114-20.

22. Trapnell C, Pachter L, Salzberg SL. TopHat: discovering splice junctions with RNA-Seq. Bioinformatics. 2009;25(9):1105-11.

23. Shen SH, Park JW, Lu ZX, Lin L, Henry MD, Wu YN, Zhou Q, Xing Y. rMATS: robust and flexible detection of differential alternative splicing from replicate RNA-Seq data. P Natl Acad Sci USA. 2014:111(51):E5593-601.

24. Kamburov A, Wierling $\mathrm{C}$, Lehrach $\mathrm{H}$, Herwig R. ConsensusPathDB-a database for integrating human functional interaction networks. Nucleic Acids Res. 2009;37:D623-8

25. Yang XA, Li JR, Lee $Y$, Lussier YA. GO-module: functional synthesis and improved interpretation of gene ontology patterns. Bioinformatics. 2011; 27(10):1444-6.

26. Szklarczyk D, Franceschini A, Wyder S, Forslund K, Heller D, Huerta-Cepas J, Simonovic M, Roth A, Santos A, Tsafou KP, et al. STRING v10: protein-protein interaction networks, integrated over the tree of life. Nucleic Acids Res. 2015:43(D1):D447-52.

27. Berman HM, Westbrook J, Feng Z, Gilliland G, Bhat TN, Weissig H, Shindyalov IN, Bourne PE. The Protein Data Bank. Nucleic Acids Res. 2000; 28(1):235-42.

28. Seeliger D, de Groot BL. Ligand docking and binding site analysis with PyMOL and autodockNina. J Comput Aid Mol Des. 2010;24(5):417-22.

29. Freed EO. HIV-1 gag proteins: diverse functions in the virus life cycle. Virol. 1998;251(1):1-15.

30. Seu L, Sabbaj S, Duverger A, Wagner F, Anderson JC, Davies E, Wolschendorf F, Willey CD, Saag MS, Goepfert P, et al. Stable phenotypi changes of the host T cells are essential to the long-term stability of latent HIV-1 infection. J Virol. 2015:89(13):6656-72.

31. Ao ZJ, Zhu R, Tan XL, Liu LS, Chen LY, Liu SP, Yao XJ. Activation of HIV-1 expression in latently infected CD4+T cells by the small molecule PKC412. Virol J. 2016;13.

32. Victoriano AF, Asamitsu K, Hibi Y, Imai K, Barzaga NG, Okamoto T. Inhibition of human immunodeficiency virus type 1 replication in latently infected cells by a novel IkappaB kinase inhibitor. Antimicrob Agents Chemother. 2006;50(2):547-55.

33. Gursoy A, Keskin O, Nussinov R. Topological properties of protein interaction networks from a structural perspective. Biochem Soc Trans. 2008, 36(Pt 6):1398-403.

34. Izumi T, lo K, Matsui M, Shirakawa K, Shinohara M, Nagai Y, Kawahara M, Kobayashi M, Kondoh $\mathrm{H}$, Misawa N, et al. HIV-1 viral infectivity factor interacts with TP53 to induce G2 cell cycle arrest and positively regulate viral replication. Proc Natl Acad Sci U S A. 2010;107(48):20798-803.

35. Ott M, Geyer M, Zhou Q. The control of HIV transcription: keeping RNA polymerase II on track. Cell Host Microbe. 2011;10(5):426-35.

36. Chou S, Upton H, Bao K, Schulze-Gahmen U, Samelson AJ, He NH, Nowak A, Lu HS, Krogan NJ, Zhou Q, et al. HIV-1 tat recruits transcription elongation factors dispersed along a flexible AFF4 scaffold. P Natl Acad Sci USA. 2013; 110(2):E123-31.

37. Hug N, Longman D, Caceres JF. Mechanism and regulation of the nonsense-mediated decay pathway. Nucleic Acids Res. 2016;44(4):1483-95. 
38. Gao G, Wu X, Zhou J, He M, He JJ, Guo D. Inhibition of HIV-1 transcription and replication by a newly identified cyclin T1 splice variant. J Biol Chem. 2013;288(20):14297-309.

39. Urano E, Miyauchi K, Ichikawa R, Futahashi Y, Komano J. Regulation of cyclin $\mathrm{T} 1$ expression and function by an alternative splice variant that skips exon 7 and contains a premature termination codon. Gene. 2012;505(1):1-8.

40. Zhou J, Gao G, Hou P, Li CM, Guo D. Regulation of the alternative splicing and function of Cyclin T1 by the serine-arginine-rich protein ASF/SF2. J Cell Biochem. 2017;118(11):4020-32

41. Abdel-Mohsen M, Kuri-Cervantes L, Grau-Exposito J, Spivak AM, Nell RA, Tomescu C, Vadrevu SK, Giron LB, Serra-Peinado C, Genesca M, et al. CD32 is expressed on cells with transcriptionally active HIV but does not enrich for HIV DNA in resting T cells. Sci Transl Med. 2018;10(437).

42. Osuna CE, Lim SY, Kublin JL, Apps R, Chen E, Mota TM, Huang SH, Ren Y, Bachtel ND, Tsibris AM, et al. Evidence that CD32a does not mark the HIV-1 latent reservoir. Nat. 2018:561(7723):E20-8.

43. Badia R, Ballana E, Castellvi M, Garcia-Vidal E, Pujantell M, Clotet B, Prado JG, Puig J, Martinez MA, Riveira-Munoz E, et al. CD32 expression is associated to Tcell activation and is not a marker of the HIV-1 reservoir. Nat Commun. 2018;9.

44. Barboric M, Peterlin BM. A new paradigm in eukaryotic biology: HIV tat and the control of transcriptional elongation. PLoS Biol. 2005;3(2):e76.

45. Johri MK, Mishra R, Chhatbar C, Unni SK, Singh SK. Tits and bits of HIV tat protein. Expert Opin Biol Ther. 2011;11(3):269-83.

46. Anand K, Schulte A, Fujinaga K, Scheffzek K, Geyer M. Cyclin box structure of the P-TEFb subunit cyclin T1 derived from a fusion complex with EIAV tat. J Mol Biol. 2007:370(5):826-36.

47. Rice AP. The HIV-1 tat protein: mechanism of action and target for HIV-1 cure strategies. Curr Pharm Des. 2017;23(28):4098-102.

48. Katz Y, Wang ET, Airoldi EM, Burge CB. Analysis and design of RNA sequencing experiments for identifying isoform regulation. Nat Methods. 2010;7(12):1009-15.

\section{Publisher's Note}

Springer Nature remains neutral with regard to jurisdictional claims in published maps and institutional affiliations.

Ready to submit your research? Choose BMC and benefit from:

- fast, convenient online submission

- thorough peer review by experienced researchers in your field

- rapid publication on acceptance

- support for research data, including large and complex data types

- gold Open Access which fosters wider collaboration and increased citations

- maximum visibility for your research: over $100 \mathrm{M}$ website views per year

At $\mathrm{BMC}$, research is always in progress.

Learn more biomedcentral.com/submissions 